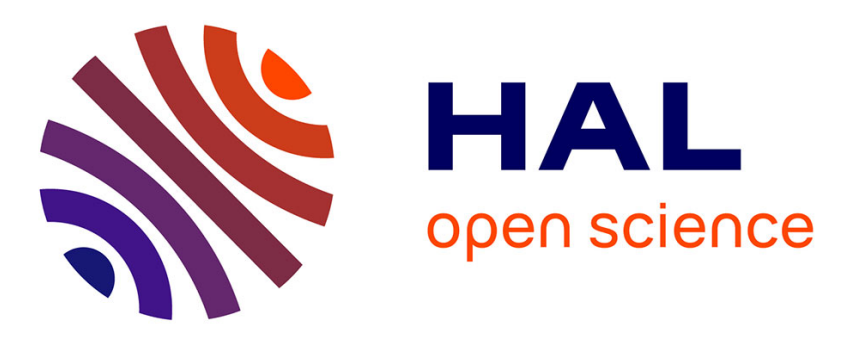

\title{
Radiotheranostic Agent \\ 64Cu-cyclam-RAFT-c(-RGDfK-)4 for Management of Peritoneal Metastasis in Ovarian Cancer
}

Zhao-Hui Jin, Atsushi B Tsuji, Mélissa Degardin, Aya Sugyo, Satoshi Obara, Hidekatsu Wakizaka, Kotaro Nagatsu, Kuan Hu, Ming-Rong Zhang, Pascal Dumy, et al.

\section{To cite this version:}

Zhao-Hui Jin, Atsushi B Tsuji, Mélissa Degardin, Aya Sugyo, Satoshi Obara, et al.. Radiotheranostic Agent 64Cu-cyclam-RAFT-c(-RGDfK-)4 for Management of Peritoneal Metastasis in Ovarian Cancer. Clinical Cancer Research, In press, 26 (23), pp.6230-6241. 10.1158/1078-0432.CCR-20-1205 . hal02943140

\author{
HAL Id: hal-02943140 \\ https://hal.science/hal-02943140
}

Submitted on 22 Sep 2020

HAL is a multi-disciplinary open access archive for the deposit and dissemination of scientific research documents, whether they are published or not. The documents may come from teaching and research institutions in France or abroad, or from public or private research centers.
L'archive ouverte pluridisciplinaire HAL, est destinée au dépôt et à la diffusion de documents scientifiques de niveau recherche, publiés ou non, émanant des établissements d'enseignement et de recherche français ou étrangers, des laboratoires publics ou privés. 


\section{Radiotheranostic Agent ${ }^{64} \mathrm{Cu}$-cyclam-RAFT-c(-RGDfK-) 4 for Management of Peritoneal Metastasis in Ovarian Cancer}

Zhao-Hui Jin ${ }^{1 *}$, Atsushi B. Tsuji ${ }^{1 *}$, Mélissa Degardin ${ }^{2}$, Aya Sugyo ${ }^{1}$, Satoshi Obara ${ }^{1}$, Hidekatsu Wakizaka ${ }^{1}$, Kotaro Nagatsu ${ }^{1}, \mathrm{Kuan}_{\mathrm{Hu}}{ }^{1}$, Ming-Rong Zhang ${ }^{1}$, Pascal Dumy ${ }^{3}$, Didier Boturyn ${ }^{2}$, and Tatsuya Higashi ${ }^{1}$

\section{Affiliations:}

${ }^{1}$ National Institute of Radiological Sciences, National Institutes for Quantum and Radiological Science and Technology, Chiba, Japan. ${ }^{2}$ Université Grenoble Alpes, CNRS, DCM, Grenoble, France. ${ }^{3}$ Institut des Biomolécules Max Mousseron, École Nationale Supérieure de Chimie de Montpellier, Université de Montpellier, Montpellier, France.

Running Title: Radiotheranostics for ovarian cancer metastasis

\section{Keywords:}

Ovarian cancer peritoneal metastases, intra- and intertumoral heterogeneity, $\alpha_{v} \beta_{3}$ integrin and tumor microenvironment, peptide-receptor radiotheranostics, ${ }^{64} \mathrm{Cu}$-labeled 
multimeric RGD peptide

\section{Financial Information}

No financial support was declared.

\section{*Corresponding Authors:}

Zhao-Hui Jin, MD, PhD

Department of Molecular Imaging and Theranostics, National Institute of Radiological

Sciences, National Institutes for Quantum and Radiological Science and Technology, Anagawa 4-9-1, Inage, Chiba 263-8555, Japan. Phone: 81-43-382-3706; Fax: 81-43-2060818; E-mail: jin.zhao-hui@.qst.go.jp

Atsushi B. Tsuji, PhD

Department of Molecular Imaging and Theranostics, National Institute of Radiological Sciences, National Institutes for Quantum and Radiological Science and Technology, Anagawa 4-9-1, Inage, Chiba 263-8555, Japan. Phone: 81-43-382-3704; Fax: 81-43-2060818; E-mail: tsuji.atsushi@qst.go.jp

\section{Disclosure of Potential Conflicts of Interest}


A.B. Tsuji reports grants from Japan Society for the Promotion of Science during the conduct of the study; grants from Japan Society for the Promotion of Science, grants from Chugai Pharmaceutical, grants from Japan Agency for Medical Research and Development, and personal fees from RIKEN outside the submitted work. No potential conflicts of interest were disclosed by the other authors.

Abstract: 250 words

Translational Relevance: 127 words

Main Text: 5438 words (from Introduction to Discussion)

Total number of references: 50

Total number of figures: 6

Total number of tables: 0

Supplementary Files:

Supplementary Figures (with Figure Legends)

Supplementary Tables

Supplementary Materials and Methods 


\section{Translational Relevance}

Despite advances in surgical cytoreduction and drug development, the 5-year survival rate for patients with ovarian cancer peritoneal metastasis (OCPM) remains as low as $<30 \%$. Although ovarian cancer cells are radiosensitive, external beam radiotherapy is rarely used to treat OCPM because of the high toxicity of wide-field irradiation. OCPMs are pathophysiologically heterogeneous tumors, highlighting the requirement for not only personalized but also tumoralized treatments in such complex context. Here, we demonstrate an all-in-one, tumor penetrating peptide-based, imagingguided, and radiotargeted strategy that offers new hope for management of OCPM with a low potential risk of radiotoxicity. We further show that simultaneous investigation of intra- and intertumoral heterogeneity in relation to the tumor microenvironment provides a basis for understanding treatment limitations and facilitates a rational combination therapy design. 


\begin{abstract}
Purpose: Ovarian cancer peritoneal metastases (OCPMs) are a pathophysiologically heterogeneous group of tumors that are rarely curable. $\alpha_{V} \beta_{3}$ Integrin $\left(\alpha_{v} \beta_{3}\right)$ is overexpressed on tumoral neovessels and frequently on ovarian cancer cells. Here, using two clinically relevant $\alpha_{v} \beta_{3}$-positive OCPM mouse models, we studied the theranostic potential of an $\alpha_{V} \beta_{3}$-specific radiopeptide, ${ }^{64} \mathrm{Cu}$-cyclam-RAFT-c(-RGDfK-) 4 $\left({ }^{64} \mathrm{Cu}\right.$-RaftRGD), and its intra- and intertumoral distribution in relation to the tumor microenvironment.
\end{abstract}

Experimental Design: $\alpha_{V} \beta_{3}$-Expressing peritoneal and subcutaneous models of ovarian carcinoma (IGR-OV1 and NIH:OVCAR-3) were established in nude mice. ${ }^{64} \mathrm{Cu}-$ RaftRGD was administered either intravenously or intraperitoneally. We performed intratumoral distribution (ITD) studies, PET/CT imaging and quantification, biodistribution assay and radiation dosimetry, and therapeutic efficacy and toxicity studies.

Results: Intraperitoneal administration was an efficient route for targeting ${ }^{64} \mathrm{Cu}-$ RaftRGD to OCPMs with excellent tumor penetration. Using the fluorescent surrogate Cy5.5-RaftRGD in our unique high-resolution multifluorescence analysis, we found that the ITD of ${ }^{64} \mathrm{Cu}$-RaftRGD was spatially distinct from but complementary to that of 
hypoxia. ${ }^{64} \mathrm{Cu}$-RaftRGD-based PET enabled clear visualization of multiple OCPM deposits and ascites; biodistribution analysis demonstrated an inverse correlation between tumor uptake and tumor size $(1.2-17.2 \mathrm{~mm}) .{ }^{64} \mathrm{Cu}-\mathrm{RaftRGD}$ at a radiotherapeutic dose (148 MBq/0.357 nmol) showed antitumor activities by inhibiting tumor cell proliferation and inducing apoptosis, and negligible toxicity.

Conclusions: Collectively, these results demonstrate the all-in-one potential of ${ }^{64} \mathrm{Cu}$-RaftRGD for imaging-guided radiotherapy of OCPM by targeting both tumoral neovessels and cancerous cells. Based on the ITD finding, we propose that pairing $\alpha_{V} \beta_{3}-$ and hypoxia-targeted radiotherapies could improve therapeutic efficacy by overcoming the heterogeneity of ITD encountered with single-agent treatments. 


\section{Introduction}

Epithelial ovarian cancer (OC) accounts for over $90 \%$ of ovarian malignancies, typically occurs in postmenopausal women, and remains the leading cause of death from gynecological malignancies (1). OC is a highly metastatic disease characterized by widely disseminated tumor depositions throughout the peritoneal cavity together with malignant ascites (2). Because of the lack of clear symptoms or an effective screening method in the early stage of the disease, over $70 \%$ of OC patients are diagnosed with peritoneal metastases (PMs) and have a 5-year survival rate of $<30 \%(1,2)$. Standard care for OCPM consists of cytoreductive surgery followed by courses of chemotherapy. Unfortunately, most patients ultimately develop recurrent chemoresistance after multiple relapses, resulting in a dismal prognosis (3). Although OC cells are radiosensitive (4), conventional external beam radiotherapy is typically not suited for OCPM because of the high toxicity of wide-field (whole-abdomen) irradiation (5). Therefore, there is a great demand for the development of new targeted therapeutic options for OCPM (6).

$\alpha_{V} \beta_{3}$ Integrin $\left(\alpha_{V} \beta_{3}\right)$ is a transmembrane cell adhesion receptor and a receptor for the well-known cyclic Arg-Gly-Asp sequence-containing pentapeptide (cRGD) ligands (7). It is overexpressed on tumoral neo-endothelial cells during angiogenesis and frequently on cancerous cells, where it regulates angiogenesis and has roles in tumor 
proliferation, invasion, and metastasis $(8,9)$. Elevated expression of $\alpha_{v} \beta_{3}$ has been reported in OC cell lines $(10,11)$ and in $\sim 80 \%$ of human OC specimens $(12,13)$. However, the use of $\alpha_{V} \beta_{3}$ inhibitors (antibody and peptide) to block the $\alpha_{V} \beta_{3}$-associated pathways has had limited effect for OC treatment (14). This has also been the case with other molecular targeted therapy studies, perhaps because multiple key pathways are involved in cancer progression and can compensate when a single pathway is blocked $(14,15)$.

Targeted radionuclide therapy (TRT) is an evolving cancer treatment modality based on systemic administration of a specific carrier (antibody, peptide, or small molecule) conjugated with a cytocidal energy-releasing radionuclide (emitting $\alpha$ or $\beta^{-}$ particles or Auger electrons) for carrier-guided irradiation of both primary and metastatic lesions (16). Peptide-based carriers may offer key advantages, such as rapid blood clearance, high tissue penetration, and low immunogenicity (17). Several groups, including our own, have demonstrated the therapeutic potential of TRT based on the cRGD ligand and $\alpha_{V} \beta_{3}$ receptor $\left(\alpha_{V} \beta_{3}\right.$-TRT) using subcutaneous (s.c.) mouse models of several cancer types (18-20), but little work has been done using the OCPM model. In 2007, Dijkgraaf et al. reported a survival-prolonging effect for the ${ }^{177}$ Lu-labeled monomeric cRGD peptide in the mouse NIH:OVCAR-3 (OVCAR-3) OCPM model (21). They started the treatment on ascites cell clusters and small tumor depositions (but 
provided no information on tumor size). An ${ }^{111}$ In-labeled counterpart was found to accumulate mainly in the periphery of the examined tumors, demonstrating the heterogeneous intratumoral distribution (ITD) of this cRGD monomer.

${ }^{64} \mathrm{Cu}$-cyclam-RAFT-c(-RGDfK-) $4\left({ }^{64} \mathrm{Cu}\right.$-RaftRGD, Fig. 1A) is a tetrameric cRGD peptide with high $\alpha_{\mathrm{v}} \beta_{3}$-binding affinity and specificity that is armed with a promising theranostic radionuclide. ${ }^{64} \mathrm{Cu}$ has a suitable half-life $(12.7 \mathrm{~h})$ and multiple decay modes, including $\beta^{+}$emission, which can be used for positron emission tomography (PET), and the combined emission of $\beta^{-}$and Auger electrons, which can be used for therapeutic irradiation (22-24). We previously demonstrated the use of ${ }^{64} \mathrm{Cu}$-RaftRGD PET to successfully visualize and quantify $\alpha_{v} \beta_{3}$ expression in mice bearing s.c. xenografts of U87MG glioblastoma and HuH-7 hepatocellular carcinoma, as well as mice bearing an orthotopic xenograft of BxPC-3 pancreatic cancer (24-27). We subsequently showed the therapeutic potential of ${ }^{64} \mathrm{Cu}$-RaftRGD and its ${ }^{67} \mathrm{Cu}\left(\beta^{-}\right)$analog in the U87MG model $(20,28)$. Here, we evaluated the utility of ${ }^{64} \mathrm{Cu}-\mathrm{RaftRGD}$ in a clinically relevant $\alpha_{v} \beta_{3^{-}}$ positive OCPM mouse model by determining the optimal route of drug administration and investigating both its PET compatibility and its therapeutic potential (in terms of radiation dosimetry, antitumor activity, and effects on normal tissues). Given the heterogeneous nature of OCPM lesions, we also studied the ITD and intertumoral 
distribution of ${ }^{64} \mathrm{Cu}-\mathrm{RaftRGD}$ in relation to selected components of the tumor microenvironment.

\section{Materials and Methods}

\section{Peptide probes}

Cyclam-RAFT-c(-RGDfK-)4 (molecular weight [MW]: 4,119.6; Fig. 1A), Cy5.5conjugated RAFT-c(-RGDfK-)4 (Cy5.5-RaftRGD; MW: 5,234.3; Cy5.5: emission maximum, 694 nm; Fig. 1A), and RAFT-c(-RGDfK-) 4 (RaftRGD; MW: 3,879.3) were synthesized as described $(22,23,29) .{ }^{64} \mathrm{CuCl}_{2}$ (radionuclide purity: $>99.9 \%$ ) and ${ }^{64} \mathrm{Cu}$ RaftRGD (maximal molar activity: $740 \mathrm{MBq} / \mathrm{nmol}$; radiochemical purity: >99\%) were prepared based on reported methods $(24,30,31)$.

\section{Cells, tumor models, and target expression}

Two human epithelial ovarian adenocarcinoma cell lines were used. IGR-OV1 was obtained through a Material Transfer Agreement (\#1-5734-18) from the National Cancer Institute Developmental Therapeutics Program Tumor Repository (Frederick, MD; 2018-May-25; authenticated by Applied Biosystems AmpFISTR Indentifier testing). OVCAR-3 was obtained directly from the American Type Culture Collection (2018- 
March-15; characterized by DNA profile and cytogenetic analysis). Cells were cultured according to the supplier's instructions. It should be stated that upon arrival (passage 0), the two lines were expanded and frozen in our laboratory (no further authentication and mycoplasma testing were performed), and early passage cells (passages 6-7 and 11-14 for IGR-OV1 and OVCAR-3, respectively) were used for all experiments.

All animal studies were approved by the Animal Ethics Committee of the National Institutes for Quantum and Radiological Science and Technology and were conducted in accordance with institutional guidelines. Four- and 7-week-old female BALB/cAJcl$n u / n u$ mice were obtained from CLEA Japan (Tokyo, Japan) for tumor-bearing and normal (non-tumor-bearing) mice studies, respectively, and acclimated for 1 week before experimentation. The mice were injected intraperitoneally (i.p.) with the designated number of IGR-OV1 or OVCAR-3 cells, and tumor development was determined by necropsy together with examination of ascites using a Leica DMI 4000B inverted microscope (Leica Microsystems, Wetzlar, Germany). For subcutaneous (s.c.) tumor reference, mice were inoculated with $5 \times 10^{6}$ IGR-OV1 or $1 \times 10^{7}$ OVCAR- 3 cells in the right flank and used for experiments at a tumor length of $6-9 \mathrm{~mm}(2-6$ weeks postxenograft).

Flow cytometry and immunohistochemistry for $\alpha_{v} \beta_{3}$ expression were performed 
using reported methods $(10,20)$, which is briefly described in the Supplementary Information.

\section{ITD of fluorescent surrogate}

Cy5.5-RaftRGD (10 nmol in $0.2 \mathrm{~mL}$ PBS) was administered intravenously (i.v., via the tail vein) or i.p. to OCPM-bearing mice and i.v. to mice bearing IGR-OV1 s.c. tumors. Mice were euthanized at $2 \mathrm{~h}$ post-injection (p.i.) and selected tumors were removed and cryosectioned (10- $\mu \mathrm{m}$ thickness) as described (26). Air-dried sections were simultaneously scanned (at $700 \mathrm{~nm}$ and $21-\mu \mathrm{m}$ resolution) using an Odyssey CLx NIR Imaging System (LI-COR Biosciences, Lincoln, NE) to visualize Cy5.5-RaftRGD distribution as described (31). Afterwards, sections were subjected to CD31 staining as described (26) with anti-CD31 (1:1,500 dilution; BD Pharmingen, San Diego, CA) and IRDye 680CW-conjuagted goat anti-rat IgG (1:200 dilution; LI-COR Biosciences) to visualize vasculature. Nuclei were counterstained with 4',6-diamidino-2-phenylindole (DAPI). Stained sections were scanned with the Odyssey as above. DAPI staining was imaged using a Keyence BZ-X710 fluorescence microscope (Keyence, Osaka, Japan). It should be noted that the fluorescence intensity of Cy5.5-RaftRGD was remarkably weakened during the immunostaining process, and thus did not interfere with the 
detection of CD31 staining.

\section{Colocalization of Cy5.5-RaftRGD and ${ }^{64} \mathrm{Cu}-\mathrm{RaftRGD}$}

OCPM-bearing mice received sequential i.v. or i.p. co-administration of ${ }^{64} \mathrm{Cu}-$ RaftRGD (37 MBq/0.1 nmol in $0.2 \mathrm{~mL}$ normal saline with 1\% Tween 80 [NS/Tw80], the vehicle for ${ }^{64} \mathrm{Cu}$-RaftRGD (20)) followed by $10 \mathrm{nmol}$ Cy5.5-RaftRGD at an interval of 10-20 min to avoid competitive receptor blocking. Three hours after the first injection, mice were euthanized. Cryosections of selected tumors with adjacent small intestine were sequentially subjected to autoradiography as described (refs. $(26,31)$; briefly described in the Supplementary method), Odyssey scanning as described above, and hematoxylin and eosin (HE) staining. Adjacent sections were subjected to CD31/DAPI staining as described above and imaged using the Keyence microscopy. Tumors of untreated mice were subjected to Odyssey scanning and HE staining to discriminate background fluorescence (autofluorescence). ${ }^{64} \mathrm{CuCl}_{2}$ (37 MBq in $0.2 \mathrm{~mL}$ PBS) was injected either i.v. or i.p. into another set of OCPM-bearing mice, and selected tumors at $3 \mathrm{~h}$ p.i. were subjected to autoradiography and CD31/DAPI/HE staining.

ITD of Cy5.5-RaftRGD in relation to the tumor microenvironment 
OCPM-bearing mice received concurrent i.p. co-administration of $10 \mathrm{nmol} \mathrm{Cy5.5-}$ RaftRGD and 1.5 mg pimonidazole (Hypoxyprobe-1 Omni Kit; Hypoxyprobe, Burlington, MA) and euthanized at $2 \mathrm{~h}$ p.i. Tumor cryosections were sequentially subjected to: (I) fixation with $2 \%$ paraformaldehyde and DAPI staining; (II) microscopy to visualize Cy5.5-RaftRGD distribution; (III) double staining by co-incubation with antiCD31 (1:200 dilution) and rabbit anti-pimonidazole (1:100 dilution; Hypoxyprobe-1 Omni Kit) followed by Alexa Fluor 594-conjugated goat anti-rat IgG (1:100 dilution; Invitrogen, Waltham, MA) and IRDye 800CW-conjugated goat anti-rabbit IgG (1:100 dilution; LI-COR Biosciences); and (IV) microscopy to visualize CD31 and pimonidazole adducts (Pimo) staining. Microscopic images of each section in its entirety were captured using a 20× objective on the Keyence microscope with DAPI, TRITC (for CD31 staining), Cy5.5, and $\mathrm{Cy} 7$ (for Pimo staining) filters using identical settings for resolution and capture position. On adjacent sections, $\alpha_{V} \beta_{3}$-immunohistochemistry was additionally performed for reference.

Similar methods were used in mice bearing IGR-OV1 or OVCAR-3 s.c. tumors receiving i.v. injection of Cy5.5-RaftRGD and pimonidazole. See the Supplementary Information for the detailed procedures. 


\section{PET/computerized tomography (CT) or PET/contrast-enhanced CT (CECT)}

PET, CT and CECT for the designated groups of mice were carried out according to our reported methods $(25,27)$, which is briefly described in the Supplementary Information. All scans (mouse in prone position) were displayed as decay-corrected maximum-intensity-projection (MIP)-PET images or as fused PET/CT or CECT images using Inveon Research Workplace software (version 4.0, Siemens). Quantification was performed on coronal PET images, which is briefly described in the Supplementary Information. All mice were euthanized immediately after the scans $(\sim 24 \mathrm{~h}$ p.i.), after which selected tumors and major organs were harvested for activity measurement with decay correction on a gamma counter (2480 WIZARD ${ }^{2}$, PerkinElmer, Waltham). For ascites, cells were spun down at 9,100 $g$ for $2 \mathrm{~min}$, and radioactivity was measured in the cell pellet and supernatant. Data are expressed as the percentage injected radioactivity dose $(\% \mathrm{ID})$ per gram tissue or per milliliter ascites $(\% \mathrm{ID} / \mathrm{g}$ or $\% \mathrm{ID} / \mathrm{mL}$; normalized to a mouse of 20-g body weight).

\section{Biodistribution and radiation dosimetry}

In the first experiment, normal mice ( $n=3-5 /$ group) were injected i.p. with 0.74 $\mathrm{MBq} / 0.2 \mathrm{nmol}{ }^{64} \mathrm{Cu}-\mathrm{RaftRGD}$ and euthanized at $2 \mathrm{~min}, 20 \mathrm{~min}, 1 \mathrm{~h}, 3 \mathrm{~h}, 24 \mathrm{~h}$, and $48 \mathrm{~h}$ 
p.i. Organs of interest were harvested for $\% \mathrm{ID} / \mathrm{g}$ quantification. Absorbed doses (ADs) were estimated using the residence time represented by the area under the curve of organ activity vs. time and the mean energy emitted per transition of ${ }^{64} \mathrm{Cu}\left(1.22 \times 10^{-14} \mathrm{~Gy} \mathrm{~kg}\right.$ $\left.\mathrm{Bq}^{-1} \mathrm{~s}^{-1}\right)$ as described (20).

In the second experiment, a similar assay was performed in OCPM-bearing mice ( $n=3$ /group). For a blocking experiment, mice $(n=3)$ were co-injected with a 100 -fold excess of unlabeled RaftRGD (20 nmol) and examined at $3 \mathrm{~h}$ p.i. Blood, ascites, and tumors of varying size (weight and length) were assessed.

\section{Radiotherapeutics}

OCPM-bearing mice ( $n=2$ or 3/group) were injected i.p. with NS/Tw80 (vehicle control) or ${ }^{64} \mathrm{Cu}-\mathrm{RaftRGD}(148 \mathrm{MBq} / 0.357 \mathrm{nmol})$ and euthanized after 3 days. Tumors of varying size, small intestine, and kidney were dissected and cryosectioned for HE staining and immunostaining for proliferation (Ki-67) and apoptosis (TUNEL assay). The procedures, based on our reported methods (20), are described in details in the Supplementary Information. 
Survival study ( $n=9 /$ group) and toxicity evaluation ( $n=5 /$ group) were additionally performed in OCPM-bearing and normal mice, respectively. See details in the Supplementary Information.

\section{Statistical analysis}

Quantitative data are presented as the mean $\pm \mathrm{SD}$. Student's $t$-tests (unpaired and two-tailed) were used for two-group comparisons, and a one-way ANOVA with Dunnett's test was used for multiple-group comparisons (KaleidaGraph 4.0). Correlations were determined using Pearson's correlation test (GraphPad Prism 7.04). $P<0.05$ was considered significant.

\section{Results}

\section{Tumor establishment and target distribution}

Fig. 1B demonstrated expression of $\alpha_{\mathrm{V}} \beta_{3}$ in $\sim 10 \%$ of OVCAR-3 cells and $>50 \%$ of IGR-OV1 cells. Both lines induced tumor formation when inoculated i.p. or s.c. into athymic nude mice, with IGR-OV1 displaying higher tumorigenicity. On days 52-67 after i.p. injection of $4 \times 10^{7}$ OVCAR-3 cells, no visible abdominal distention was observed, and three of the four mice (75\%) formed primarily milky and viscous ascites 
that contained large amounts of cancer cell clusters (Fig. 1C; Supplementary Fig. S1A) and small numbers (five or fewer) of tumor deposits in the peritoneal cavity (Fig. 1C). In contrast, following i.p. injection of $1 \times 10^{7}$ IGR-OV1 cells, four of four mice (100\%) developed visibly distended abdomens, copious yellowish or bloody ascites containing a high density of single cancer cells and cancer cell clusters (Fig. 1D; Supplementary Fig. S1B), and dozens of various-sized tumor lesions disseminated throughout the peritoneal cavity (Fig. 1D) within 14-18 days. Immunohistochemical staining with anti-human $\alpha_{v} \beta_{3}$ showed strong membranous staining of tumor cells in mice receiving OVCAR-3 and IGR-OV1 i.p. injections (Fig. 1C and D) or s.c. xenografts (Supplementary Fig. S2). Intratumoral heterogeneous expression of $\alpha_{v} \beta_{3}$ was also obvious, with the greatest staining in perivascular tumor cells or the periphery of tumor cell nests, where tumor cells were in contact with surrounding tissues. Based on these findings, we used mice inoculated i.p. with IGR-OV1 cells ( 18 days after xenograft) as the main OCPM model (if not specified otherwise) in the present study. These mice had the typical OCPM presentation described above, with no visible signs of compromised health.

\section{Effect of injection route on ITD of ${ }^{64} \mathrm{Cu}$-RaftRGD}

To predict the ITD of ${ }^{64} \mathrm{Cu}$-RaftRGD, we analyzed its fluorescent surrogate, 
Cy5.5-RaftRGD at $2 \mathrm{~h}$ after i.v. and i.p. injection into mice bearing IGR-OV1 OCPM (IGR-OV1-OCPM mice). Fig. 2 shows representative images of the distribution of Cy5.5-RaftRGD (Fig. 2A) and corresponding blood vessels (Fig. 2B) in entire sections of OCPM lesions (a cluster of small nodules and a single large nodule) after i.v. and i.p. injection, and reference images from an IGR-OV1 s.c. tumor after i.v. injection. In all tumors examined, there was no demonstrable difference in Cy5.5-RaftRGD uptake between the tumor center and periphery, which thus resulted in a whole-tumor binding pattern. Under comparable conditions (in terms of tumor size and vasculature), peritoneal tumors labeled with an i.p. injection showed a generally higher overall uptake than those labeled with an i.v. injection. Comparison of large nodules demonstrated that the only notable difference in Cy5.5-RaftRGD distribution between i.v. and i.p. injection routes was a higher uptake at the tumor periphery following i.p. injection. This is mostly attributable to direct penetration of the probe from the surrounding ascites. Comparison of the i.v. injection between peritoneal and s.c. tumors indicated that the lower uptake related to peritoneal tumors might be due at least in part to the compromised vasculature that develops in the peritoneal cavity as compared with the highly vascularized s.c. xenograft (Fig. 2B). 


\section{Colocalization of Cy5.5-RaftRGD and ${ }^{64} \mathrm{Cu}-\mathrm{RaftRGD}$}

Intratumoral colocalization of Cy5.5-RaftRGD and ${ }^{64} \mathrm{Cu}-\mathrm{RaftRGD}$ was validated by NIR scanning and autoradiography of the same whole-tumor sections at $\sim 3 \mathrm{~h}$ after i.v. or i.p. co-administration to IGR-OV1-OCPM mice. Fig. 3 shows representative images of the well-matched and overlapping distribution of Cy5.5-RaftRGD and ${ }^{64} \mathrm{Cu}-\mathrm{RaftRGD}$ in clusters of small tumors after i.v. or i.p. injection. In contrast to i.v. injection, i.p. injection led to uptake of ${ }^{64} \mathrm{Cu} / \mathrm{Cy} 5.5-\mathrm{RaftRGD}$ in avascular or poorly vascularized tumor lesions. The ITD of free ${ }^{64} \mathrm{CuCl}_{2}$ (Supplementary Fig. S3), injected either i.v. or i.p., demonstrated poor tumor selectivity (compared with adjacent intestinal tissues) in the present OCPM model. Moreover, i.p.-administered ${ }^{64} \mathrm{CuCl}_{2}$ resulted in accumulation of radioactivity that was predominantly in the peripheral rim of the tumor, forming a ringlike appearance.

\section{ITD of Cy5.5-RaftRGD in relation to the tumor microenvironment}

Next, the ITD of Cy5.5-RaftRGD (representing ${ }^{64} \mathrm{Cu}$-RaftRGD) was analyzed in relation to CD31-stained blood vessels and regions of Pimo-stained hypoxic cells by highresolution multifluorescence microscopy in tumor sections at $2 \mathrm{~h}$ after co-administration of the probes to IGR-OV1-OCPM mice. Fig. $\mathbf{4 A - J}$ shows a representative OCPM section 
exhibiting separate and merged images of nuclei, Cy5.5-RaftRGD, microvessels, and regions of hypoxia. In Fig. 4D-F, regions of partial overlap (yellow) between Cy5.5RaftRGD (red; Fig. 4B) and microvessels (green; Fig. 4C) denote $\alpha_{v} \beta_{3}$-overexpressing neovessels. In Fig. 4H-J and Supplementary Fig. S4B, merged images of Cy5.5RaftRGD and hypoxia (green; Fig. 4G) clearly demonstrate the discordant but complementary distributions of Cy5.5-RaftRGD and hypoxia. The overlapping signal at the tumor periphery is most likely attributable to direct penetration of the two i.p.-injected probes from the surrounding ascites. Similar results were obtained in IGR-OV1 and OVCAR-3 s.c. tumor models (Fig. 4K-N and more details in Supplementary Fig. S5).

\section{Clearance and accumulation of ${ }^{64} \mathrm{Cu}-\mathrm{RaftRGD}$ by PET/CT or PET/CECT imaging}

Fig. 5 shows representative whole-body MIP-PET and magnified abdominal MIPPET/CT (or CECT) images after i.v. or i.p. injection of $0.2 \mathrm{nmol}{ }^{64} \mathrm{Cu}-\mathrm{RaftRGD}(\sim 18.5$ or $37 \mathrm{MBq}$ ) in tumor-bearing and normal mice. Here and below, this 0.2-nmol dose was supplemented by the addition of unlabeled cyclam-RAFT-c(-RGDfK-) 4 to be the equivalent of a 148-MBq therapy plan. The i.v. injection in IGR-OV1-OCPM mice resulted in rapid blood clearance and notable renal excretion as early as 5 min after injection, with generally low accumulation of abdominal radioactivity and tumors that 
were barely detectable (Fig. 5A, D, and E). In i.p.-treated IGR-OV1-OCPM mice (Fig. 5B, F, and G) and normal mice (Fig. 5C, H, and I), high levels of radioactivity accumulated throughout the abdominal area within the first $1 \mathrm{~h}$ p.i. After 3.5 and $24 \mathrm{~h}$ p.i., this noticeable background declined significantly, allowing delineation of accumulation within the renal excretion system. Meanwhile, multiple focal regions of high radioactivity uptake were detected only in the abdominal cavity of tumor-bearing mice, indicating the sites of metastases. These findings were validated by the results of quantitative PET (Supplementary Fig. S6A), post-PET biodistribution (Supplementary Fig. S6B and Supplementary Table S1), and time-course biodistribution given in the following section. Additional PET/CT or PET/CECT of i.p.-treated mice bearing OVCAR-3 OCPM (OVCAR-3-OCPM mice) showed strong radioactivity signals in regions of ascites highaccumulation (associated with the body position; Fig. 5J, K, and L), with high values of $11.2 \pm 1.99$ and $8.3 \pm 2.05 \% \mathrm{ID} / \mathrm{g}_{\max }($ maximal $\% \mathrm{ID} / \mathrm{g}$ ) at 3.5 and $24 \mathrm{~h}$ p.i., respectively (Supplementary Fig. S7A). Post-PET biodistribution ( 24 h p.i.) revealed $98.8 \pm 0.15 \%$ of ascites radioactivity attributed from cancer cell clusters (as seen in Supplementary Fig. S1A) with a high uptake of $16.2 \pm 2.92 \%$ ID/g (Supplementary Fig. S7B and S7C).

\section{Biodistribution data and radiation dosimetry of ${ }^{64} \mathrm{Cu}$-RaftRGD}


The biodistribution results of i.p.-administered ${ }^{64} \mathrm{Cu}$-RaftRGD (i.p. ${ }^{64} \mathrm{Cu}$ RaftRGD; 0.74 MBq/0.2 nmol) in normal mice are summarized in Supplementary Fig. S8 and Supplementary Table S2. In the blood, radioactivity peaked $(0.59 \pm 0.09 \% \mathrm{ID} / \mathrm{g})$ at $1 \mathrm{~h}$ p.i. and dropped to a negligible level $(0.04 \pm 0.01 \% \mathrm{ID} / \mathrm{g})$ at $24 \mathrm{~h}$ p.i. The kidneys had the highest uptake $(24.8 \pm 2.14$ and $18.2 \pm 1.59 \% \mathrm{ID} / \mathrm{g}$ at 3 and $24 \mathrm{~h}$ p.i., respectively), indicating prominent renal excretion. The estimated mean ADs in blood and major abdominal organs are also presented in Supplementary Fig. S8 (human dosimetry is appended in Supplementary Table S4 for reference). The kidneys received the highest dose (24.6 Gy when injected with $\left.148 \mathrm{MBq} / 0.2 \mathrm{nmol}{ }^{64} \mathrm{Cu}-\mathrm{RaftRGD}\right)$. Doses to nonabdominal organs (such as heart and lungs) can be inferred from our previous data for i.v.-administered ${ }^{64} \mathrm{Cu}$-RaftRGD (20), as the PET (Fig. 5A-C) and post-PET biodistribution (Supplementary Fig. S6B) analyses demonstrated similarly low accumulation levels of radioactivity in these organs after i.v. and i.p. injection.

The results of blood and ascites obtained from IGR-OV1-OCPM mice are summarized in Supplementary Figs. S9 and S10A-S10G, respectively, and those of tumors are shown in Fig. 6A and B and Supplementary Figs. S11 and S12. There was little to no difference in blood radioactivity levels and kinetics between tumor-bearing and normal mice (Supplementary Fig. S9). The concentration of radioactivity in ascites 
(Supplementary Fig. S10A) declined steadily over time, from $40.6 \pm 10.7$ and $31.4 \pm$ $3.6 \% \mathrm{ID} / \mathrm{mL}$ at 2 and $20 \mathrm{~min}$ p.i., respectively, to $17.4 \pm 1.9,6.3 \pm 0.2$, and $0.89 \pm$ $0.12 \% \mathrm{ID} / \mathrm{mL}$ at 1,3 , and $24 \mathrm{~h}$ p.i., respectively. This result is consistent with the changes in abdominal radioactivity as visualized by PET (Fig. 5B). Uptake in the cell pellet fraction from ascites peaked at $3 \mathrm{~h}$ p.i. $(13.0 \pm 2.2 \% \mathrm{ID} / \mathrm{g}$; Supplementary Fig. S10F) and decreased gradually to $5.1 \pm 0.47 \% \mathrm{ID} / \mathrm{g}$ at $48 \mathrm{~h}$ p.i. Because the ascites cell pellets of IGR-OV1-OCPM mice also contained large numbers of blood cells (Supplementary Fig. S1B), these data can be considered underestimates of the level of uptake by the ascites cancer cells. Uptake of radioactivity was then evaluated in 162 tumors (nine tumors of varying size per mouse; Supplementary Fig. S11) that had a $r$ value of 0.920 between tumor weight and length $(P<0.0001$; Supplementary Fig. S12). Similar to the cancer cell clusters in ascites, tumor deposits had a peak uptake at $3 \mathrm{~h}$ p.i. The mean peak uptake of the smallest tumors $(\leq 2.6 \mathrm{~mm} ; 10 \pm 1.9 \% \mathrm{ID} / \mathrm{g})$ was nearly two times that of the largest tumors $(\geq 10 \mathrm{~mm} ; 5.3 \pm 1.0 \% \mathrm{ID} / \mathrm{g}, P=0.0003)$. Fig. 6A shows scatter plots and fitted curves of tumor uptake vs. length at multiple time points, revealing a significant inverse correlation for each time point. Size-specific tumor uptake values were predicted at the highest values of $r$ using the inverse regression relationship expressed by the equation $y=\frac{a}{x}+b \quad$ (Supplementary Table S3). Next, size-dependent estimated ADs were 
obtained (Fig. 6B). Finally, co-injection with an excess of unlabeled RaftRGD resulted in a marked decrease in the uptake of ${ }^{64} \mathrm{Cu}$-RaftRGD by both tumor nodules (irrespective of size; Fig. 6A; Supplementary Fig. S11) and ascites cell pellets (Supplementary Fig. S10G), revealing an $\alpha_{V} \beta_{3}$-specific binding mechanism.

\section{Therapeutic potential and heterogeneity}

Fig. 6C and $\mathbf{D}$ show the effects of i.p. injection of ${ }^{64} \mathrm{Cu}-\mathrm{RaftRGD}(148 \mathrm{MBq} / 0.357$ nmol) on proliferation and apoptosis in tumors of IGR-OV1-OCPM mice, respectively. The peptide dose of $0.357 \mathrm{nmol}$ was slightly higher than the planned dose $(0.2 \mathrm{nmol})$ due to the molar activity of $415 \mathrm{MBq} / \mathrm{nmol}$ obtained from the radiolabeling. It should be noted that, as RaftRGD itself has no antitumor activity even at a dose of $4 \mathrm{nmol}$ (28), a peptide control was not used in this therapy study. Tumors from both vehicle- and ${ }^{64} \mathrm{Cu}-$ RaftRGD-treated mice ranged from a few hundreds of micrometers to $>10 \mathrm{~mm}$ in length and demonstrated highly varied Ki-67 proliferation indices (percentage of cells positive for Ki-67) and apoptotic indices (percentage of apoptotic cells). To minimize the effect of tumor size on changes in proliferation and apoptosis, tumors were categorized into three groups based on tumor length and number of tumors (Fig. 6C and D): $<1 \mathrm{~mm}, 1-4$ $\mathrm{mm}$, and $>4 \mathrm{~mm} .{ }^{64} \mathrm{Cu}$-RaftRGD reduced proliferation by $47.9 \%, 62.8 \%, 40.9 \%$, and 
27.9\% and increased apoptosis by 3.2-, 4.8-, 3.0-, and 2.7-fold in comparisons among all lesions and lesions $<1 \mathrm{~mm}, 1-4 \mathrm{~mm}$, and $>4 \mathrm{~mm}$, respectively. Supplementary Fig. S13 shows the results of proliferation/morphology studies on small intestine and kidney. One major finding is that the proliferation index in kidney was lower in ${ }^{64} \mathrm{Cu}-\mathrm{RaftRGD}$-treated mice than in control mice $(0.26 \pm 0.07 \%$ vs. $1.72 \pm 0.15 \%, P=0.0068)$.

The long-term antitumor activity of the single-dose of $148 \mathrm{MBq} / 0.357 \mathrm{nmol}$ i.p. ${ }^{64} \mathrm{Cu}-\mathrm{RaftRGD}$ was revealed by the survival study (summarized in Supplementary Fig. S14A-S14F). Ascites formation was markedly suppressed (Supplementary Fig. S14E), and in line with the Ki-67 and apoptosis analyses described above, the smaller-sized tumors (like those along the intestine) appeared to be more efficiently treated (Supplementary Fig. S14A and S14F).

The results of toxicity evaluation of $148 \mathrm{MBq} / 0.357 \mathrm{nmol}$ i.p. $\cdot{ }^{64} \mathrm{Cu}-\mathrm{RaftRGD}$ in normal mice as long as 60 days after administration are given in Supplementary Figs. S15A-S15J and S16A-S16C. No severe toxicity occurred as the most noticeable findings include a mild transient decrease of blood counts and slightly elevated levels of plasma creatinine on day 60 .

\section{Discussion}


OCPM presents with a pathophysiologically heterogeneous group of tumors, typically with the co-existence of ascites cancer cell clusters and solid tumors of varying size disseminated throughout the peritoneal cavity. To be effective and informative, a new TRT strategy for OCPM should be developed based on an in-depth understanding of the relevant intratumoral heterogeneity (IntraTH) and intertumoral heterogeneity (InterTH). The present study shows the theranostic potential of an $\alpha_{v} \beta_{3}$-targeted multimeric cRGD peptide-based radiopharmaceutical agent in clinically relevant mouse models of OCPM (IGR-OV1 and OVCAR-3), along with analysis of the IntraTH and InterTH of both drug uptake and therapeutic effect. Importantly, it also demonstrates a spatially complementary relationship between the ITD of the $\alpha_{\mathrm{V}} \beta_{3}$-targeting agent and regions of tumor hypoxia. Determining the optimal administration route (i.v. or i.p. injection) to achieve efficient targeting to PMs has been a long-standing issue that needs to be addressed $(21,32)$. Because i.p. injection can deliver drug to the tumor not only via the blood circulation (through absorption by mesenteric and lymphatic vessels) but also via direct penetration, it can result in increased drug concentrations in the tumor $(33,34)$. However, the outcome will also depend on the collective effects of a number of other factors, such as the efficiency of drug diffusion inside the peritoneal cavity upon injection, the capability of the drug to penetrate the tumor, the metabolic stability of the drug in ascites 
fluid, and the extent of drug binding to ascites or tissue proteins. The present study demonstrates that the tumor-targeting efficiency of ${ }^{64} \mathrm{Cu}-\mathrm{RaftRGD}$ in OCPM-bearing mice was markedly influenced by the route of administration. A direct comparison showed that the i.p. route produced high levels of tumor uptake (Supplementary Fig. S6) in contrast to the less-efficient i.v. route, enabling PET visualization of multiple regions of metastases (both of solid tumors and malignant ascites) in the peritoneal cavity (Fig. 5). Moreover, the observed whole-tumor binding patterns of i.p.-administered Cy5.5/ ${ }^{64} \mathrm{Cu}-$ RaftRGD observed within a few hours after injection (2-3 h p.i.; Figs. 2-4) indicate that RaftRGD-based probes have an excellent ability to penetrate tumors.

$\alpha_{V} \beta_{3}$-Specific tumor uptake of i.p. ${ }^{64} \mathrm{Cu}$-RaftRGD was validated by co-injection of an excess of unlabeled parent RaftRGD peptide, which resulted in a significant decrease in accumulation of radioactivity in both solid tumor deposits (Fig. 6A and Supplementary Fig. S11) and ascites cell pellets (Supplementary Fig. S10E and S10G). Moreover, the ITD of free ${ }^{64} \mathrm{CuCl}_{2}$ administered i.p. or i.v. showed poor tumor selectivity, with a predominant ring-like distribution at the tumor periphery after i.p. injection (Supplementary Fig. S3), indicating poor tumor penetration. The distinct differences between the binding patterns of ${ }^{64} \mathrm{Cu}-\mathrm{RaftRGD}$ and ${ }^{64} \mathrm{CuCl}_{2}$ demonstrate the stability of ${ }^{64} \mathrm{Cu}-\mathrm{RaftRGD}$ in ascites fluid, with no major release of ${ }^{64} \mathrm{Cu}$ from the probe. Notably, 
the distribution of ${ }^{64} \mathrm{CuCl}_{2}$ indicates that it was incapable, at least in the present case, of specifically targeting PMs, although some recent preclinical studies have demonstrated its theranostic potential for several other cancer types, including melanoma, glioblastoma, and prostate cancer as reviewed by Gutfilen et al. (35).

We observed an inverse correlation between tumor uptake of i.p. ${ }^{64} \mathrm{Cu}$-RaftRGD and tumor size $(1.2-17.2 \mathrm{~mm}$ ) at all examined time points ( $2 \mathrm{~min}$ to $48 \mathrm{~h}$ p.i.; Fig. 6A), consistent with other reports on different radiocompounds and tumor models (36-38). This indicates an InterTH in which small tumors received proportionally higher radiation doses. This size effect can be affected by a number of other factors, such as changing levels of target density or activity or the degree of tumor vascularity, hypoxia, and necrosis. In the present case, hypoxia appeared to be closely related to ${ }^{64} \mathrm{Cu}$-RaftRGD tumor uptake, based on the spatially complementary ITD patterns of compound and hypoxia (described further below) and an increase in the severity of hypoxia with increasing tumor size (Supplementary Fig. S17A). Based on the correlation between i.p. ${ }^{64} \mathrm{Cu}$-RaftRGD tumor uptake and tumor size, we developed inverse regression equations for each time point p.i. (Supplementary Table S3), allowing us to predict tumor uptake based on tumor size and hence calculate an estimated size-dependent AD (Fig. 6B). 
InterTH was also found with regard to the antitumor activity of i.p. ${ }^{64} \mathrm{Cu}$-RaftRGD against OCPMs of various sizes. Antitumor activity was evaluated based on the ability of i.p.-administered compound to inhibit tumor proliferation (Fig. 6C) and induce apoptosis (Fig. 6D), leading to a marked suppression of ascites formation (Supplementary Fig. S14E) and tumor growth (Supplementary Fig. S14F). A rough association was seen between tumor size and therapeutic response. At ADs of $>28.2,28.2-8.8$, and $<8.8$ Gy to tumors $<1 \mathrm{~mm}, 1-4 \mathrm{~mm}$, and $>4 \mathrm{~mm}$ (data derived from Fig. 6B), we observed a $62.8 \%$, $40.9 \%$, and $27.9 \%$ reduction in proliferation index and a 4.8-, 3.0-, and 2.7-fold increase in apoptotic index, respectively. Given the heterogenic effects of this therapy, our findings suggest approaches for optimizing treatment parameters based on the extent of metastasis (such as the tumor size).

Tumor heterogeneity, including that of drug distribution, is an inevitable obstacle limiting treatment efficacy $(39,40)$. In TRT, the heterogeneous ITD of radiocompounds, due at least in part to the intrinsically heterogeneous ITD of targets (e.g., different antigen expression levels among cancer cells) as well as the influence of the tumor microenvironment, can cause non-uniform delivery of cytocidal radiation throughout the tumor mass, resulting in a non-uniform therapeutic response. In the present study, we investigated the ITD of ${ }^{64} \mathrm{Cu}$-RaftRGD in relation to two important features of the solid 
tumor microenvironment: tumor vasculature and hypoxia (41). Using the surrogate probe Cy5.5-RaftRGD in our unique high-resolution multifluorescence analysis, we found a spatially discordant but complementary relationship between the ITD of Cy5.5-RaftRGD (in regions of angiogenesis and $\alpha_{V} \beta_{3}$-positive cancerous cells) and regions of hypoxia (where $\alpha_{V} \beta_{3}$ expression itself can be found positive as shown in Supplementary Fig. S4C) in the OCPM model as well as s.c. IGR-OV1 and OVCAR-3 models (Fig. 4). This striking finding may lay out a rationale for pairing $\alpha_{v} \beta_{3^{-}}$and hypoxia-targeted TRT agents to tackle the obstacle of heterogeneity encountered by single TRT agents. Indeed, we recently reported that the combined use of ${ }^{64} \mathrm{Cu}$-RaftRGD and ${ }^{64} \mathrm{Cu}$-diacetyl-bis $\left(\mathrm{N}^{4}\right.$ methylthiosemicarbazone) $\left({ }^{64} \mathrm{Cu}-\mathrm{ATSM}\right.$, a supposed tracer for hypoxia metabolism) in U87MG s.c. xenografts improves the antitumor effect as compared with ${ }^{64} \mathrm{Cu}$-RaftRGD alone, owing to a more uniform ITD of radioactivity (31).

The kidneys are dose-limiting organs for radiotherapy. Here, in mice receiving $148 \mathrm{MBq}$ i.p. ${ }^{64} \mathrm{Cu}$-RaftRGD, the kidneys had an estimated mean AD of 24.6 Gy. Recent peptide-based studies $(42,43)$ comparing the nephrotoxicity of ${ }^{90} \mathrm{Y}$ - and ${ }^{177} \mathrm{Lu}-$ radiolabeled somatostatin analogues propose the renal dose limits of 24 Gy for ${ }^{90} \mathrm{Y}$ and a higher value of $28 \mathrm{~Gy}$ for ${ }^{177} \mathrm{Lu}$ (due to a shorter range $\beta^{-}$particles, $2 \mathrm{~mm}$ maximum than ${ }^{90} \mathrm{Y}, \sim 11.3 \mathrm{~mm}$ maximum, causing less damage to nearby tissue). Since ${ }^{64} \mathrm{Cu}$ has a similar 
$\beta^{-}$particle range $(2.5 \mathrm{~mm}$ maximum, ref. 44$)$ with ${ }^{177} \mathrm{Lu}$, it is reasonable to postulate a renal dose limit of $\sim 28$ Gy for ${ }^{64} \mathrm{Cu}$-RaftRGD. In fact, the 60-day assessment demonstrates no evidence of severe toxicity in the $148-\mathrm{MBq}$-treated mice (Supplementary Figs. S15 and S16). On the other hand, several approaches have been introduced to prevent renal damage from high ADs of radiation (44). We previously showed that introducing Gelofusine (succinylated gelatin) and L-lysine (positively charged basic amino acid) into the i.v. injectate of ${ }^{64} \mathrm{Cu}-\mathrm{RaftRGD}$ caused $30-50 \%$ reduction in mouse renal radioactivity levels $(20,25)$. However such addition to the i.p. injectate of ${ }^{64} \mathrm{Cu}$-RaftRGD produced only a $19.6 \%$ reduction (obtained from a preliminary study shown in Supplementary Fig. S18A-S18C). An optimization of the dosing regimens of these renoprotective agents is hence required.

In conclusion, i.p. ${ }^{64} \mathrm{Cu}-\mathrm{RaftRGD}$ exhibits excellent tumor penetration capability and can be specifically targeted to all forms of OCPM, including ascites cancer cells, tiny avascular lesions, and vascularized tumors of varying size. It is applicable for PET visualization and quantification of $\alpha_{v} \beta_{3}$-positive OCPM and shows promise for $\alpha_{V} \beta_{3}$ TRT of OCPM with a low potential risk of radiotoxicity. It is hence a promising all-inone radiotheranostic agent for OCPM management and worth further study. The spatially complementary ITD patterns of ${ }^{64} \mathrm{Cu}$-RaftRGD and hypoxia suggest that pairing $\alpha_{V} \beta_{3}$ - 
and hypoxia-targeted TRT is a possible approach to tackle the problem of heterogeneous delivery of cytocidal radiation that is encountered with single TRT agents. Importantly, such combined TRT is also suitable for sub-mm sized peritoneal tumor deposits that can contain significant hypoxic fractions as reported (45) and shown in Supplementary Fig. S17B-S17D. In the future, when the use of $\alpha$-emitting radionuclides becomes practical, incorporating these agents in a paired TRT strategy would greatly improve treatment effects, as the high linear energy transfer of $\alpha$ particles induces highly potent cell-killing while overcoming the limited tissue pathlength of $\alpha$ particles-e.g., 40-100 $\mu \mathrm{m}$ for $\alpha$ particles vs. $950-1,400 \mu \mathrm{m}$ for ${ }^{64} \mathrm{Cu}(46,47) .{ }^{225} \mathrm{Ac}$ and its decay product ${ }^{213} \mathrm{Bi}$ are pure $\alpha$-emitters of interest in ovarian cancer therapy $(48,49)$. Given the facts that ${ }^{225} \mathrm{Ac}$ can be stably attached to a cRGD peptide by using a bifunctional chelator DOTA (50), and RaftRGD can be conjugated with DOTA (19), the radiolabeling of RaftRGD with ${ }^{225}$ Ac would be feasible.

\section{Authors' Contributions}

Conception and design: Z.-H. Jin, A.B. Tsuji, D. Boturyn, P. Dumy, T. Higashi

Development of methodology: Z.-H. Jin, A.B. Tsuji, M. Degardin, S. Obara

Acquisition of data (provided animals, acquired and managed patients, provided 
facilities, etc.): Z.-H. Jin, A.B. Tsuji, M. Degardin, A. Sugyo, H. Wakizaka, K. Nagatsu, M.-R. Zhang, K. Hu

Analysis and interpretation of data (e.g., statistical analysis, biostatistics, computational analysis): Z.-H. Jin, A.B. Tsuji, M. Degardin, S. Obara

Writing, review, and/or revision of the manuscript: Z.-H. Jin, A.B. Tsuji, M. Degardin, D. Boturyn, P. Dumy, T. Higashi

Administrative, technical, or material support (i.e., reporting or organizing data, constructing databases): A. Sugyo, H. Wakizaka, K. Nagatsu, M.-R. Zhang, K. Hu Study supervision: A.B. Tsuji, T. Higashi

\section{Acknowledgments}

This work was supported in part by JSPS KAKENHI Grant Number JP18K07776 (Japan) and the Agence Nationale de la Recherche (ANR-17-EURE-0003, France). The authors M. Degardin and D. Boturyn wish to acknowledge support from the ICMG Chemistry Nanobio Platform (Grenoble, France), on which the peptide synthesis was performed, and the Labex ARCANE and CBH-EUR-GS (ANR-17-EURE-0003). The authors thank the Department of Advanced Nuclear Medicine Sciences and the Cyclotron Operation Section of National Institute of Radiological Sciences (Chiba, Japan) for 
supplying ${ }^{64} \mathrm{Cu}$. We are grateful to Dr. J.-F. Gourvest (Aventis, France) for kindly providing HEK293( $\left.\beta_{1}\right)$ and HEK293( $\left.\beta_{3}\right)$ cells, and to Dr. J.-L. Coll (INSERM U1209, Grenoble) for early effort of providing IGR-OV1 cells. 


\section{References}

1. Lheureux S, Gourley C, Vergote I, Oza AM. Epithelial ovarian cancer. Lancet 2019;393:1240-53.

2. Yeung TL, Leung CS, Yip KP, Au Yeung CL, Wong ST, Mok SC. Cellular and molecular processes in ovarian cancer metastasis. a review in the theme: cell and molecular processes in cancer metastasis. Am J Physiol Cell Physiol 2015;309:C444-56.

3. Pokhriyal R, Hariprasad R, Kumar L, Hariprasad G. Chemotherapy resistance in advanced ovarian cancer patients. Biomark Cancer 2019;11:1-19.

4. Slotman BJ, Karim AB, Rao BR. Ovarian cancer: radiation sensitivity in vitro. Radiother Oncol 1990;19:323-7.

5. Fields EC, McGuire WP, Lin L, Temkin SM. Radiation treatment in women with ovarian cancer: past, present, and future. Front Oncol 2017;7:177.

6. Lokadasan R, James FV, Narayanan G, Prabhakaran PK. Targeted agents in epithelial ovarian cancer: review on emerging therapies and future developments. Ecancermedicalscience 2016;10:626.

7. Haubner R, Gratias R, Diefenbach B, Goodman SL, Jonczyk A, Kessler H. Structural and functional aspects of RGD-containing cyclic pentapeptides as highly potent and selective integrin $\alpha_{v} \beta_{3}$ antagonists. J Am Chem Soc 1996;118:7461-72.

8. Jin H, Varner J. Integrins: roles in cancer development and as treatment targets. Br J Cancer 2004;90:561-5.

9. Desgrosellier JS, Cheresh DA. Integrins in cancer: biological implications and therapeutic opportunities. Nat Rev Cancer 2010;10:9-22. 
10. Jin ZH, Josserand V, Razkin J, Garanger E, Boturyn D, Favrot MC, et al. Noninvasive optical imaging of ovarian metastases using Cy5-labeled RAFT-c(RGDfK-)4. Mol Imaging 2006;5:188-97.

11. Landen CN, Kim TJ, Lin YG, Merritt WM, Kamat AA, Han LY, et al. Tumorselective response to antibody-mediated targeting of $\alpha_{V} \beta_{3}$ integrin in ovarian cancer. Neoplasia 2008;10:1259-67.

12. Wang Y, Liu J, Lin B, Wang C, Li Q, Liu S, et al. Study on the expression and clinical significances of lewis y antigen and integrin $\alpha_{V}, \beta_{3}$ in epithelial ovarian tumors. Int J Mol Sci 2011;12:3409-21.

13. Villegas-Pineda JC, Garibay-Cerdenares OL, Hernandez-Ramirez VI, GallardoRincon D, Cantu de Leon D, Perez-Montiel-Gomez MD, et al. Integrins and haptoglobin: molecules overexpressed in ovarian cancer. Pathol Res Pract 2015;211:973-81.

14. Kobayashi M, Sawada K, Kimura T. Potential of integrin inhibitors for treating ovarian cancer: a literature review. Cancers (Basel) 2017;9:83.

15. Friedman R. Drug resistance in cancer: molecular evolution and compensatory proliferation. Oncotarget 2016;7:11746-55.

16. Malcolm J, Falzone N, Lee BQ, Vallis KA. Targeted radionuclide therapy: new advances for improvement of patient management and response. Cancers (Basel) 2019;11:268.

17. Fani M, Maecke HR, Okarvi SM. Radiolabeled peptides: valuable tools for the detection and treatment of cancer. Theranostics 2012;2:481-501.

18. Liu Z, Wang F, Chen X. Integrin targeted delivery of radiotherapeutics. Theranostics 2011;1:201-10. 
19. Bozon-Petitprin A, Bacot S, Gauchez AS, Ahmadi M, Bourre JC, Marti-Batlle D, et al. Targeted radionuclide therapy with RAFT-RGD radiolabelled with ${ }^{90} \mathrm{Y}$ or ${ }^{177} \mathrm{Lu}$ in a mouse model of $\alpha_{v} \beta_{3}$-expressing tumours. Eur $\mathrm{J}$ Nucl Med Mol Imaging 2015;42:252-63.

20. Jin ZH, Furukawa T, Degardin M, Sugyo A, Tsuji AB, Yamasaki T, et al. $\alpha_{v} \beta_{3}$ Integrin-targeted radionuclide therapy with ${ }^{64} \mathrm{Cu}$-cyclam-RAFT-c(-RGDfK-)4. Mol Cancer Ther 2016;15:2076-85.

21. Dijkgraaf I, Kruijtzer JA, Frielink C, Corstens FH, Oyen WJ, Liskamp RM, et al. $\alpha_{v} \beta_{3}$ Integrin-targeting of intraperitoneally growing tumors with a radiolabeled RGD peptide. Int J Cancer 2007;120:605-10.

22. Boturyn D, Coll JL, Garanger E, Favrot MC, Dumy P. Template assembled cyclopeptides as multimeric system for integrin targeting and endocytosis. J Am Chem Soc 2004;126:5730-9.

23. Galibert M, Jin ZH, Furukawa T, Fukumura T, Saga T, Fujibayashi Y, et al. RGDcyclam conjugate: synthesis and potential application for positron emission tomography. Bioorg Med Chem Lett 2010;20:5422-5.

24. Jin ZH, Furukawa T, Galibert M, Boturyn D, Coll JL, Fukumura T, et al. Noninvasive visualization and quantification of tumor $\alpha_{v} \beta_{3}$ integrin expression using a novel positron emission tomography probe, ${ }^{64} \mathrm{Cu}$-cyclam-RAFT-c(RGDfK-)4. Nucl Med Biol 2011;38:529-40.

25. Jin ZH, Furukawa T, Sogawa C, Claron M, Aung W, Tsuji AB, et al. PET imaging and biodistribution analysis of the effects of succinylated gelatin combined with L-lysine on renal uptake and retention of ${ }^{64} \mathrm{Cu}$-cyclam-RAFT-c(-RGDfK-) 4 in vivo. Eur J Pharm Biopharm 2014;86:478-86. 
26. Jin ZH, Furukawa T, Claron M, Boturyn D, Coll JL, Fukumura T, et al. Positron emission tomography imaging of tumor angiogenesis and monitoring of antiangiogenic efficacy using the novel tetrameric peptide probe ${ }^{64} \mathrm{Cu}$-cyclamRAFT-c(-RGDfK-)4. Angiogenesis 2012;15:569-80.

27. Aung W, Jin ZH, Furukawa T, Claron M, Boturyn D, Sogawa C, et al. Micropositron emission tomography/contrast-enhanced computed tomography imaging of orthotopic pancreatic tumor-bearing mice using the $\alpha_{v} \beta_{3}$ integrin tracer ${ }^{64} \mathrm{Cu}$ labeled cyclam-RAFT-c(-RGDfK-)4. Mol Imaging 2013;12:376-87.

28. Jin $\mathrm{ZH}$, Furukawa T, Ohya T, Degardin M, Sugyo A, Tsuji AB, et al. ${ }^{67} \mathrm{Cu}-$ Radiolabeling of a multimeric RGD peptide for $\alpha_{v} \beta_{3}$ integrin-targeted radionuclide therapy: stability, therapeutic efficacy, and safety studies in mice. Nucl Med Commun 2017;38:347-55.

29. Jin ZH, Josserand V, Foillard S, Boturyn D, Dumy P, Favrot MC, et al. In vivo optical imaging of integrin $\alpha_{V}-\beta_{3}$ in mice using multivalent or monovalent $\mathrm{cRGD}$ targeting vectors. Mol Cancer 2007;6:41.

30. Ohya T, Nagatsu K, Suzuki H, Fukada M, Minegishi K, Hanyu M, et al. Efficient preparation of high-quality ${ }^{64} \mathrm{Cu}$ for routine use. Nucl Med Biol 2016;43:685-91.

31. Jin ZH, Tsuji AB, Degardin M, Sugyo A, Yoshii Y, Nagatsu K, et al. Uniform intratumoral distribution of radioactivity produced using two different radioagents, ${ }^{64} \mathrm{Cu}$-cyclam-RAFT-c(-RGDfK-) 4 and ${ }^{64} \mathrm{Cu}$-ATSM, improves therapeutic efficacy in a small animal tumor model. EJNMMI Res 2018;8:54.

32. Dou S, Smith M, Wang Y, Rusckowski M, Liu G. Intraperitoneal injection is not always a suitable alternative to intravenous injection for radiotherapy. Cancer Biother Radiopharm 2013;28:335-42. 
33. Bajaj G, Yeo Y. Drug delivery systems for intraperitoneal therapy. Pharm Res $2010 ; 27: 735-8$.

34. Chaudhary K, Haddadin S, Nistala R, Papageorgio C. Intraperitoneal drug therapy: an advantage. Curr Clin Pharmacol 2010;5:82-8.

35. Gutfilen B, Souza SA, Valentini G. Copper-64: a real theranostic agent. Drug Des Devel Ther 2018;12:3235-45.

36. Hagan PL, Halpern SE, Dillman RO, Shawler DL, Johnson DE, Chen A, et al. Tumor size: effect on monoclonal antibody uptake in tumor models. J Nucl Med $1986 ; 27: 422-7$.

37. Harrington KJ, Rowlinson-Busza G, Syrigos KN, Abra RM, Uster PS, Peters AM, et al. Influence of tumour size on uptake of ${ }^{111}$ In-DTPA-labelled pegylated liposomes in a human tumour xenograft model. Br J Cancer 2000;83:684-8.

38. Schoffelen R, van der Graaf WT, Sharkey RM, Franssen GM, McBride WJ, Chang $\mathrm{CH}$, et al. Pretargeted immuno-PET of CEA-expressing intraperitoneal human colonic tumor xenografts: a new sensitive detection method. EJNMMI Res 2012;2:5.

39. Dagogo-Jack I, Shaw AT. Tumour heterogeneity and resistance to cancer therapies. Nat Rev Clin Oncol 2018;15:81-94.

40. Garattini S, Fuso Nerini I, D'Incalci M. Not only tumor but also therapy heterogeneity. Ann Oncol 2018;29:13-19.

41. Multhoff G, Radons J, Vaupel P. Critical role of aberrant angiogenesis in the development of tumor hypoxia and associated radioresistance. Cancers (Basel) $2014 ; 6: 813-28$.

42. Svensson J, Molne J, Forssell-Aronsson E, Konijnenberg M, Bernhardt P. 
Nephrotoxicity profiles and threshold dose values for $\left[{ }^{177} \mathrm{Lu}\right]$-DOTATATE in nude mice. Nucl Med Biol 2012;39:756-62.

43. Bergsma H, Konijnenberg MW, van der Zwan WA, Kam BL, Teunissen JJ, Kooij PP, et al. Nephrotoxicity after PRRT with ${ }^{177}$ Lu-DOTA-octreotate. Eur J Nucl Med Mol Imaging 2016;43:1802-11.

44. Cremonesi M, Ferrari M, Bodei L, Tosi G, Paganelli G. Dosimetry in peptide radionuclide receptor therapy: a review. J Nucl Med 2006;47:1467-75.

45. Li XF, Carlin S, Urano M, Russell J, Ling CC, O'Donoghue JA. Visualization of hypoxia in microscopic tumors by immunofluorescent microscopy. Cancer Res 2007;67:7646-53.

46. Poty S, Francesconi LC, McDevitt MR, Morris MJ, Lewis JS. $\alpha$-Emitters for radiotherapy: from basic radiochemistry to clinical studies-part 1. J Nucl Med 2018;59:878-84.

47. Johnsen AM, Heidrich BJ, Durrant CB, Bascom AJ, Unlu K. Reactor production of ${ }^{64} \mathrm{Cu}$ and ${ }^{67} \mathrm{Cu}$ using enriched zinc target material. J Radioanal Nucl Chem 2015;305:61-71.

48. Borchardt PE, Yuan RR, Miederer M, McDevitt MR, Scheinberg DA. Targeted actinium-225 in vivo generators for therapy of ovarian cancer. Cancer Res 2003;63:5084-90.

49. Vallon M, Seidl C, Blechert B, Li Z, Gilbertz KP, Baumgart A, et al. Enhanced efficacy of combined ${ }^{213} \mathrm{Bi}-\mathrm{DTPA}-\mathrm{F} 3$ and paclitaxel therapy of peritoneal carcinomatosis is mediated by enhanced induction of apoptosis and G2/M phase arrest. Eur J Nucl Med Mol Imaging 2012;39:1886-97.

50. Pandya DN, Hantgan R, Budzevich MM, Kock ND, Morse DL, Batista I, et al. 
Preliminary therapy evaluation of ${ }^{225}$ Ac-DOTA-c(RGDyK) demonstrates that Cerenkov radiation derived from ${ }^{225}$ Ac daughter decay can be detected by optical imaging for in vivo tumor visualization. Theranostics 2016;6:698-709. 


\section{Figure legends}

Figure 1. Molecular probes and tumor models. A, Structures of ${ }^{64} \mathrm{Cu}-\mathrm{RaftRGD}$ and its fluorescent surrogate Cy5.5-RaftRGD. RAFT, regioselectively addressable functionalized template. B, Flow cytometric analysis of $\alpha_{v} \beta_{3}$ expression in OVCAR-3 and IGR-OV1 cells. HEK293( $\left.\beta_{1}\right)$ and HEK293( $\left.\beta_{3}\right)$ cells were used as negative and positive control cells, respectively; BKG, background control (cells incubated with antibody diluent); IgG1, mouse IgG1 isotype control. Quantitative data indicate the percentage of stained cells from three or five experiments. C and D, Representative images of human OCPM models. Left panel of $(\mathbf{C})$ : Mouse euthanized on day 52 after i.p. injection of OVCAR-3 cells $\left(4 \times 10^{7}\right.$ cells $)$ presenting milky and viscous ascites, a tumor nodule localized in the sub-hepatic space (arrowhead), and large amounts of ascites cancer cells (syringe: collected ascites; tube bottom: whitish cell pellet). Left panel of (D): Mouse euthanized on day 18 after i.p. injection of IGR-OV1 cells $\left(1 \times 10^{7}\right.$ cells $)$ presenting multiple various-sized tumors (arrowheads) throughout the peritoneal cavity, massive amounts of bloody ascites, and large amounts of ascites cancer cells (syringe: collected ascites; tube bottom: whitish cell pellet). Right panels of (C and D): Immunohistochemical staining of peritoneal tumors using mouse anti-human $\alpha_{v} \beta_{3}$ (dark brown). BKG, background control (tumors stained with antibody diluent); IgG1, mouse IgG1 isotype control.

Figure 2. ITD of Cy5.5-RaftRGD at $2 \mathrm{~h}$ after i.v. and i.p. injection to OCPM-bearing mice. A, Whole-tumor sections showing distribution of Cy5.5-RaftRGD (red) administered i.v. (top) vs. i.p. (bottom) in IGR-OV1 PMs consisting of a cluster of small nodules (arrows) and a large nodule. The distribution of probe administered i.v. in the s.c. 
model was used a reference for the peritoneal model. B, Corresponding vasculature in the same sections shown in (A). Magenta, CD31-stained microvessels; blue, DAPI-stained nuclei.

Figure 3. Intratumoral colocalization of Cy5.5-RaftRGD and ${ }^{64} \mathrm{Cu}-\mathrm{RaftRGD}$ at $\sim 3 \mathrm{~h}$ after i.v. and i.p. co-administration to IGR-OV1-OCPM mice. Entire sections of a cluster of small tumor nodules with adjacent small intestine were examined. Autoradiography of ${ }^{64} \mathrm{Cu}-$ RaftRGD (green), Odyssey scanning (700 nm) of Cy5.5-RaftRGD (red), and HE staining were sequentially performed on the same sections. Microvascular staining (antiCD31, magenta) was done on adjacent sections. Yellow, red/green overlay; blue, nuclei stained with DAPI. Regions of interest are marked by solid or dashed circles, within which microvessels are shown in magnified images. The Odyssey scanning of samples from untreated mice at $700 \mathrm{~nm}$ shows that the predominant source of autofluorescence is in the intestinal contents.

Figure 4. Spatial relationship of intratumoral Cy5.5-RaftRGD to the tumor microenvironment at $2 \mathrm{~h}$ after co-administration with pimonidazole to tumor-bearing mice. A-J, A representative IGR-OV1 OCPM lesion labeled with an i.p. injection. All fluorescence microscopic images are from a single whole-tumor section showing DAPIstained nuclei (A; blue), the distribution of Cy5.5-RaftRGD (B; red), CD31-stained microvessels (C; green), and Pimo-stained hypoxia (G; green). D, merged image of (B) and $(\mathbf{C}) ; \mathbf{E}$, merged image of $(\mathbf{A}),(\mathbf{B})$, and $(\mathbf{C}) ; \mathbf{F}$, magnified image of inset (dotted region) in (E); G, Pimo staining (green); H, merged image of (B) and (G); I, merged image of (A), (B), and (G); J, magnified image of inset (dotted region) in (I), the same region as 
indicated in (E). Magenta, Cy5.5-RaftRGD fluorescence in (E) and (I); Yellow, red/green overlay. K-N, IGR-OV1 (K and L) or OVCAR-3 (M and $\mathbf{N}$ ) s.c. tumors labeled with an i.v. injection. The merged images for each tumor are from a single whole-tumor section subjected to Odyssey scanning for Cy5.5-RaftRGD (at $700 \mathrm{~nm}$, magenta), CD31-stained microvessels (at $700 \mathrm{~nm}$, green), and Pimo-stained hypoxia (at $800 \mathrm{~nm}$, green). Blue, microscopic images of DAPI-stained nuclei; yellow, magenta/green overlay.

Figure 5. PET/CT or PET/CECT imaging of OCPM-bearing mice after i.v. or i.p. injection of $0.2 \mathrm{nmol}{ }^{64} \mathrm{Cu}$-RaftRGD. IGR-OV1-OCPM mice ( $n=3 /$ group) were injected i.v. (A, D, E) or i.p. (B, F, G) with ${ }^{64} \mathrm{Cu}$-RaftRGD $(\sim 18.5 \mathrm{MBq})$; normal mice $(n=3)$ were injected i.p. at the same dose $(\mathbf{C}, \mathbf{H}, \mathbf{I})$. An additional group of OVCAR-3-OCPM mice $(n=5)$ were injected i.p. with ${ }^{64} \mathrm{Cu}-\operatorname{RaftRGD}(\sim 37 \mathrm{MBq} ; \mathbf{J}, \mathbf{K}, \mathbf{L})$. Dynamic imaging was performed for $5 \mathrm{~min}(')$ per scan from 0 to $60 \mathrm{~min}$ p.i., and 30-min static scans were performed at 3.5 and $24 \mathrm{~h}$ p.i. (except a 10-min scan for OVCAR-3 mice at $3.5 \mathrm{~h}$ ). A-C, Representative whole-body MIP-PET images at 0-24 h p.i. D-K, Representative magnified abdominal MIP-PET/CT fused images at 3.5 and 24 h p.i. L, Representative magnified abdominal MIP-PET, CECT, and MIP-PET/CECT images (coronal) at $24 \mathrm{~h}$ p.i. $B$, bladder; $H$, heart; $K$, kidney; $L$, liver; $T$, tumor; $A$, ascites. Scale in $(\mathbf{C}): 1 \mathrm{~cm}(\mathbf{A}-\mathbf{C})$; scale in $(\mathbf{K}): 1 \mathrm{~cm}(\mathbf{D}-\mathbf{K})$; scale in $(\mathbf{L}): 1 \mathrm{~cm}$.

Figure 6. Tumor uptake, estimated ADs, and anti-proliferative and apoptotic activity of ${ }^{64} \mathrm{Cu}-\mathrm{RaftRGD}$. A and B, IGR-OV1-OCPM mice ( $n=3$ /group) were injected i.p. with $0.74 \mathrm{MBq} / 0.2 \mathrm{nmol}{ }^{64} \mathrm{Cu}$-RaftRGD; nine tumors per mouse were examined at the designated time points. A, Scatter plots and inverse regression fitting of correlation 
between tumor uptake and tumor length. Open circles represent blocking with an excess of unlabeled parent peptide (RaftRGD) at $3 \mathrm{~h}$ p.i. $* * *, * * * P<0.05,0.01$, and 0.0001 , respectively. B, Tumor AD (mGy/MBq and Gy [148 MBq]) vs. tumor length. C and D, IGR-OV1-OCPM mice were injected i.p. with $148 \mathrm{MBq} / 0.357 \mathrm{nmol}{ }^{64} \mathrm{Cu}-\mathrm{RaftRGD}$ (TRT; $n=2$ ) or vehicle control (Cont, NS/Tw80; $n=3$ ). Tumors of varying size were examined at day 3 p.i. Upper panels: Representative images of Ki-67 fluorescence staining $(\mathbf{C}$; green) and TUNEL staining (D; dark brown) of tumors. Lower panels: Corresponding quantitative analysis of Ki-67 staining (percentage of positively stained cells) or TUNEL staining (percentage of apoptotic cells). Nuclei were stained with DAPI (C; blue) or methyl green (D; light green). All tumor lesions ( $n=45$ and 58 for control and ${ }^{64} \mathrm{Cu}$-RaftRGD-treated groups, respectively) were categorized according to tumor length: $<1 \mathrm{~mm}, n=19$ and $25 ; 1-4 \mathrm{~mm}, n=22$ and $29 ;>4 \mathrm{~mm}, n=4$ and 4 for control and ${ }^{64} \mathrm{Cu}-\mathrm{RaftRGD}$-treated groups, respectively. $*, * *, * * *, * * * * P<0.05,0.01,0.001$, and 0.0001 , respectively. 


\section{Figure 1}

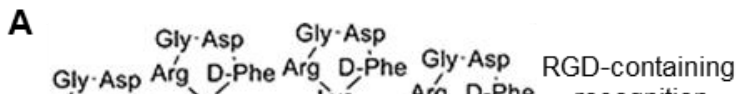

Gly·Asp Gly-Asp
Gly-Asp Aŕg D-Phe Arg D-Phe Gly-Asp RGD-containing Árg D-Phe Lys Lys Arg D-Phe recognition

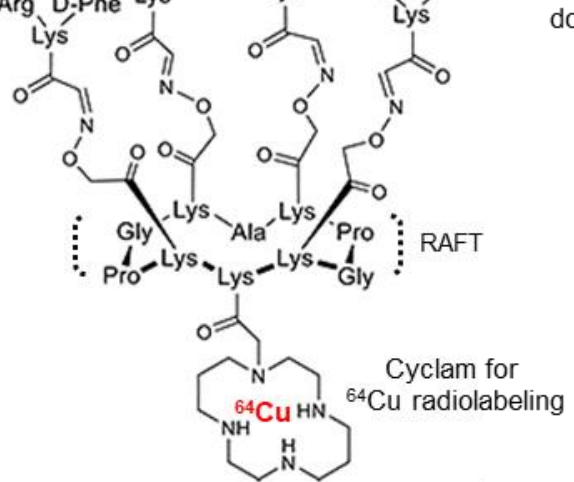

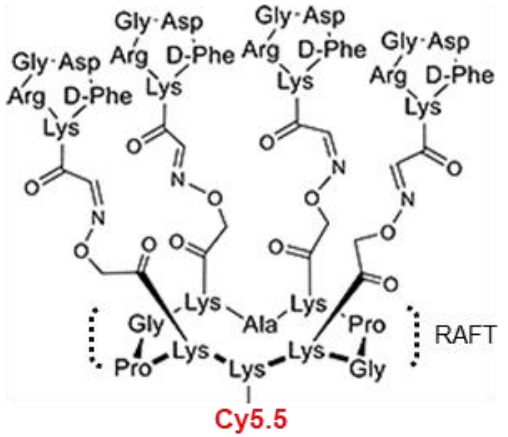

For optical imaging
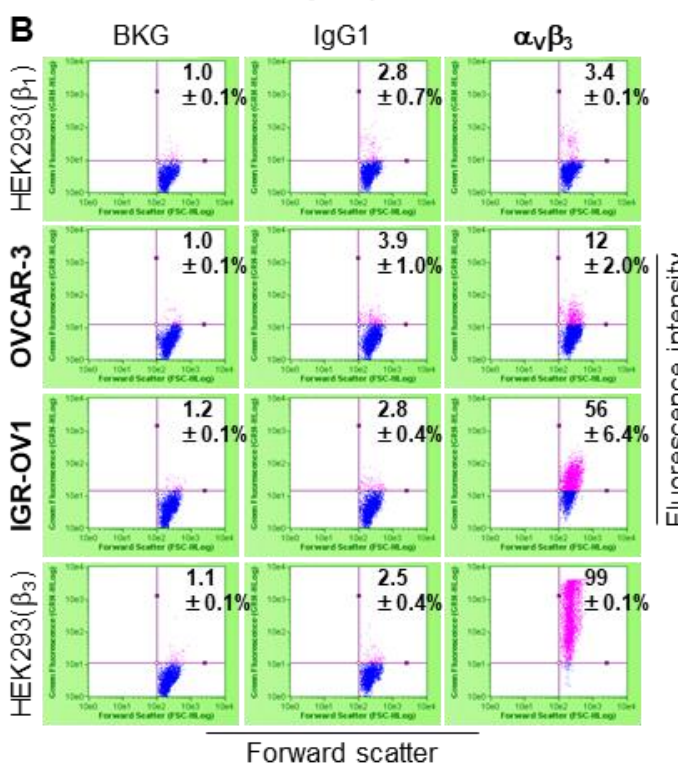

C

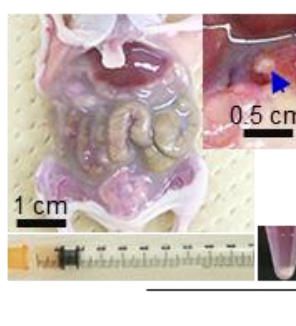

D

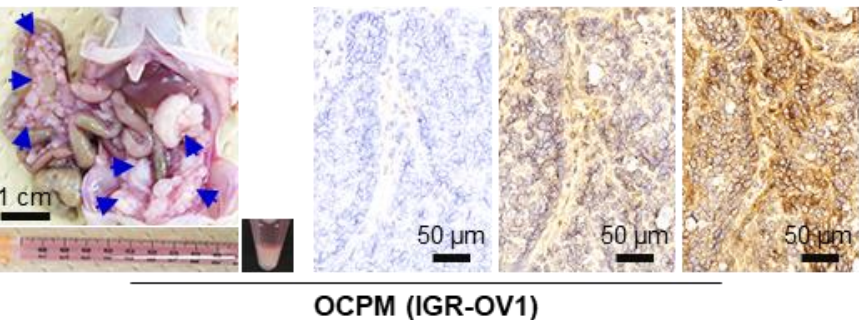


Figure 2

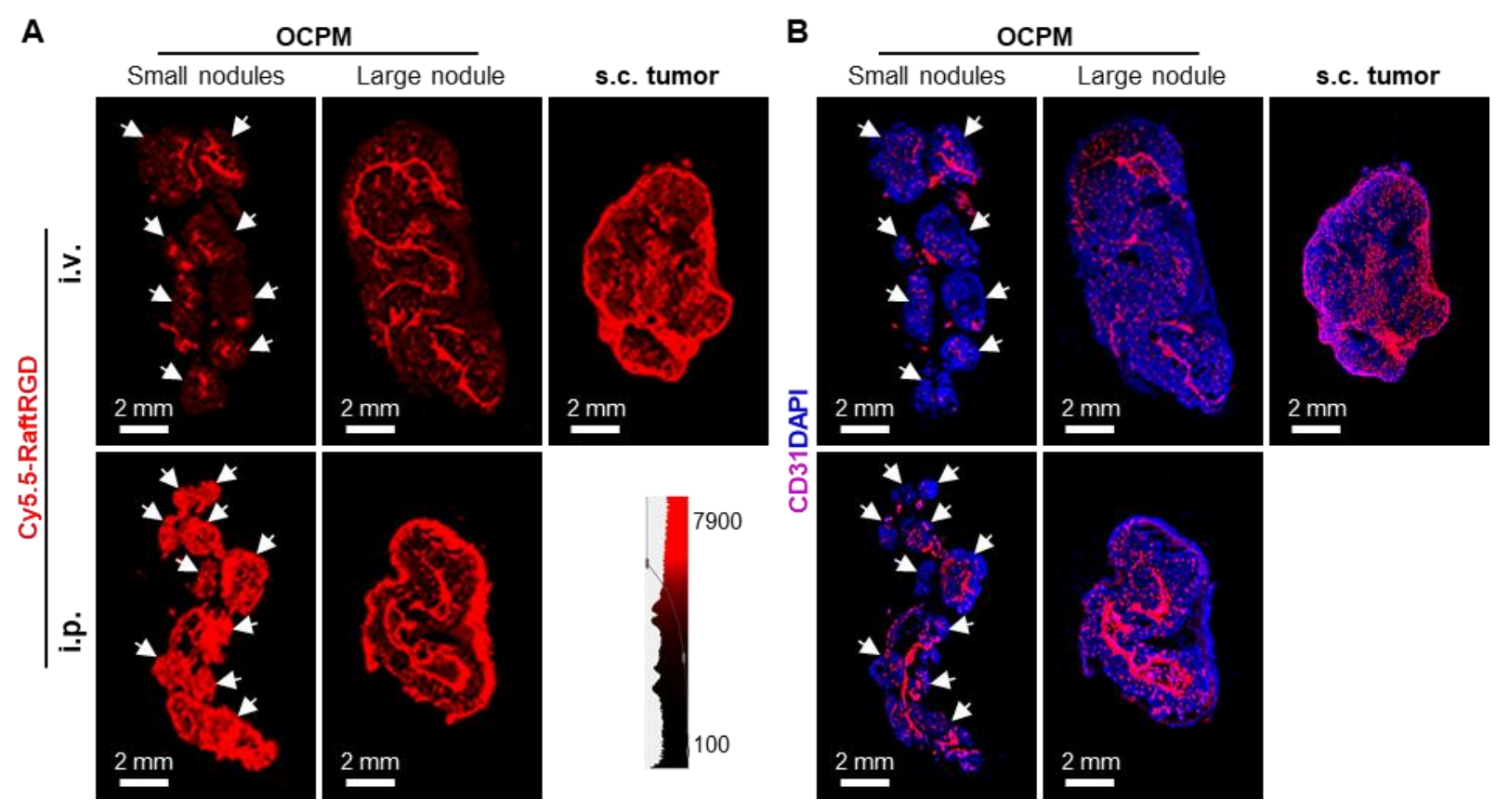


Figure 3

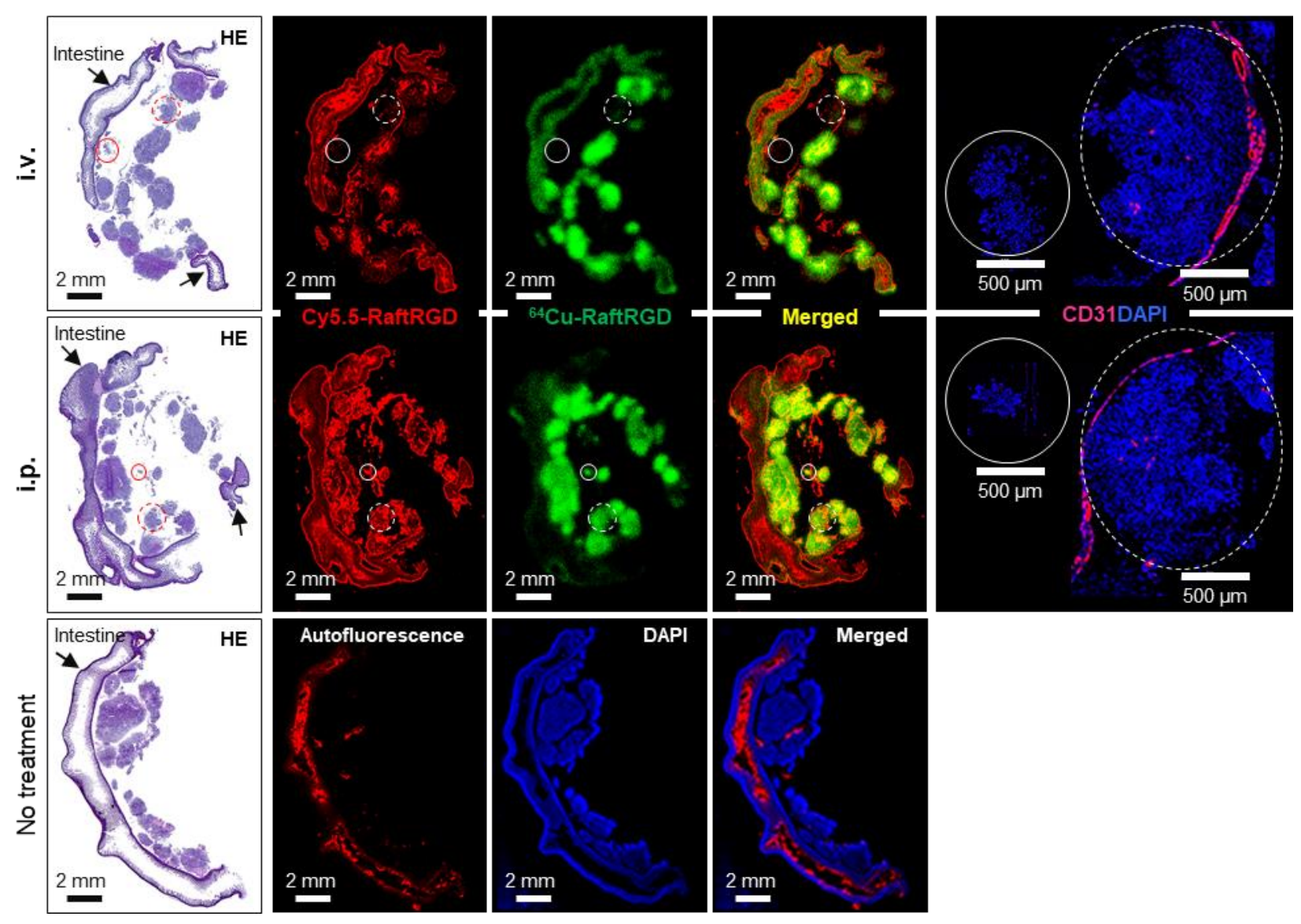


Figure 4

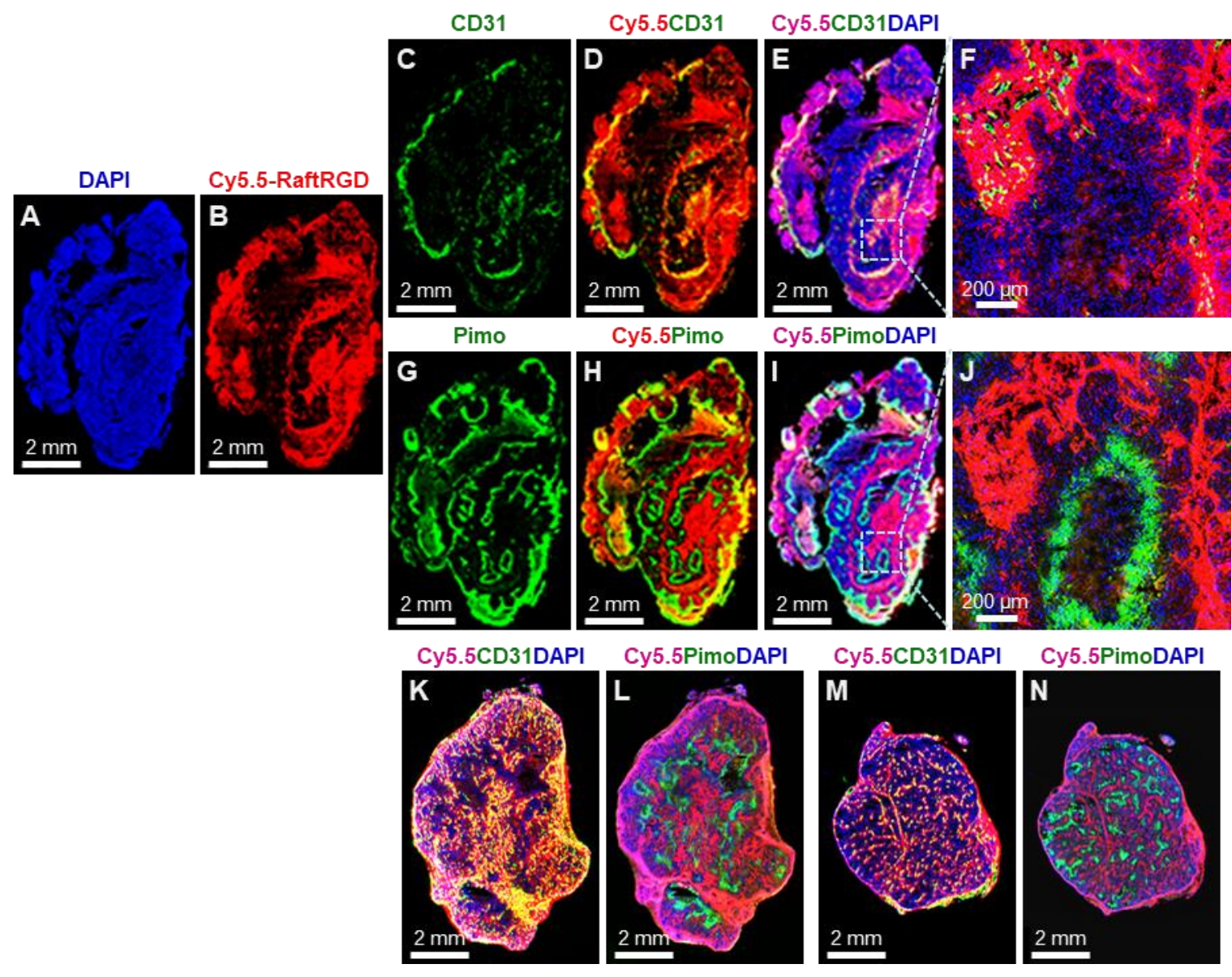


Figure 5
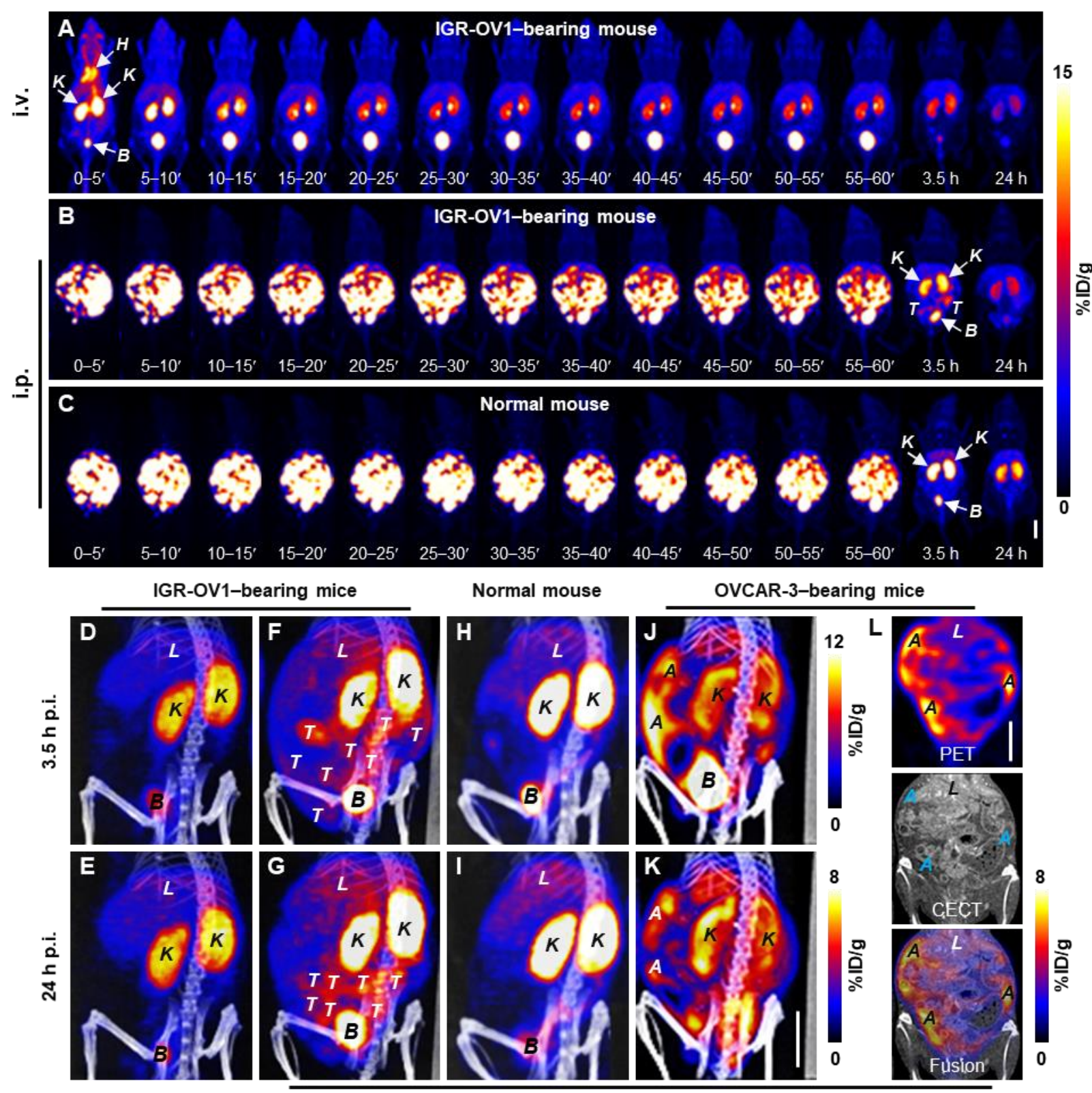

Normal mouse OVCAR-3-bearing mice
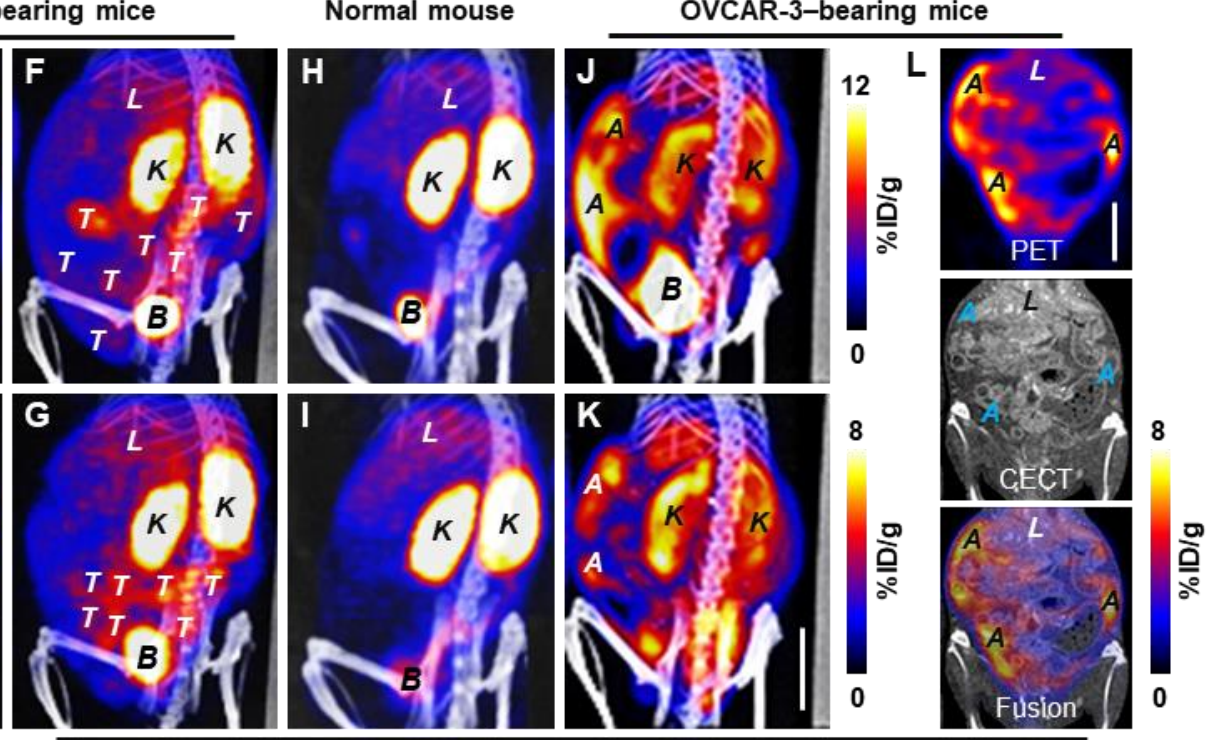

i.v.

i.p. 


\section{Figure 6}
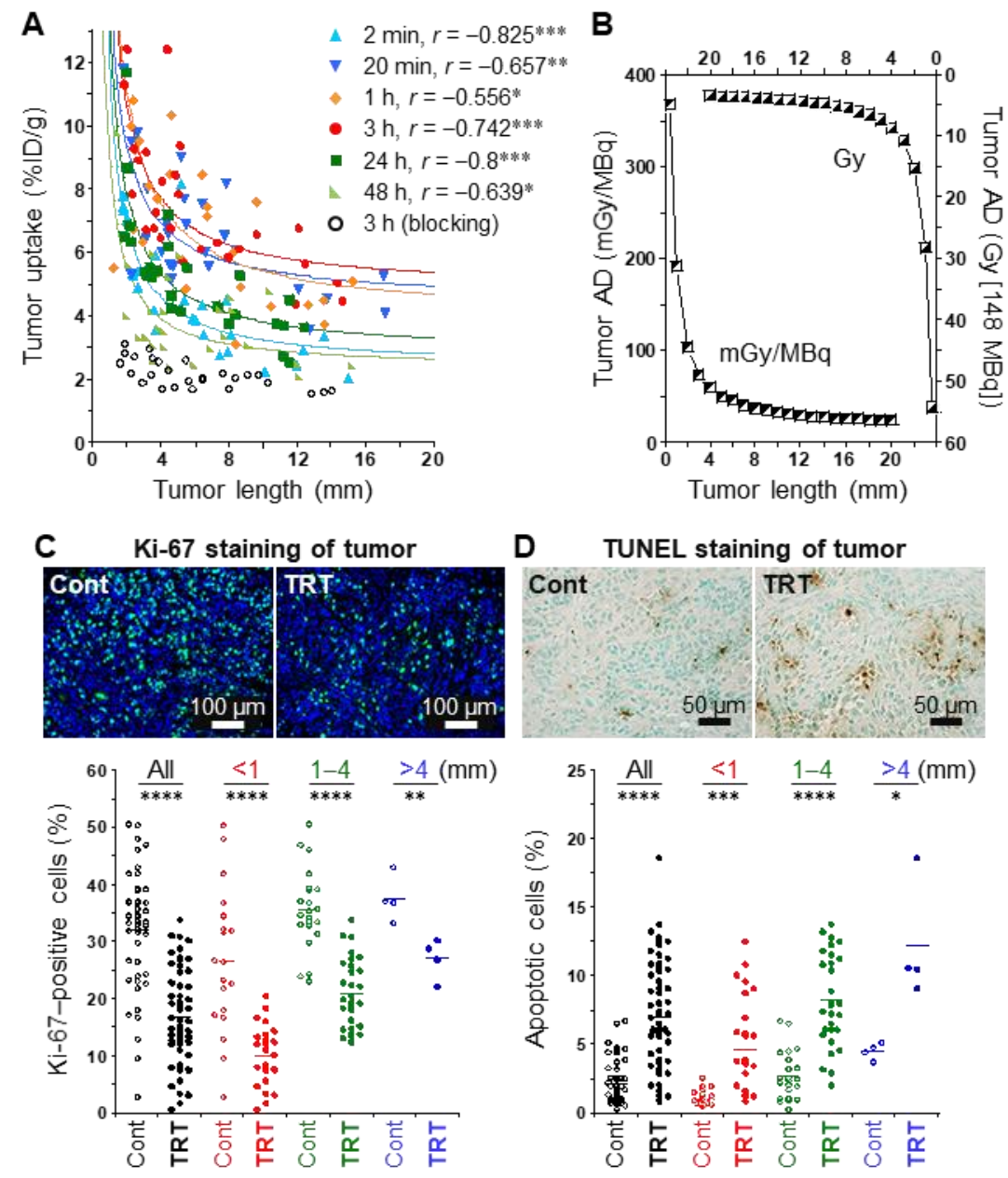